\title{
Persistent Control of a Superconducting Qubit by Stroboscopic Measurement Feedback
}

\author{
P. Campagne-Ibarcq, ${ }^{1}$ E. Flurin, ${ }^{1}$ N. Roch,${ }^{1}$ D. Darson,,${ }^{1}$ P. Morfin,,${ }^{1}$ M. Mirrahimi, ${ }^{2}$ \\ M. H. Devoret, ${ }^{3}$ F. Mallet, ${ }^{1}$ and B. Huard ${ }^{1}$ \\ ${ }^{1}$ Laboratoire Pierre Aigrain, Ecole Normale Supérieure, CNRS (UMR 8551), Université Pierre et Marie Curie, \\ Université Denis Diderot, 24 rue Lhomond, 75231 Paris Cedex 05, France \\ ${ }^{2}$ INRIA Paris-Rocquencourt, Domaine de Voluceau, Boîte Postale 105, 78153 Le Chesnay Cedex, France \\ ${ }^{3}$ Department of Applied Physics, Yale University, Post Office Box 208284, New Haven, Connecticut 06520-8284, USA
}

(Received 14 February 2013; published 29 May 2013)

\begin{abstract}
Making a system state follow a prescribed trajectory despite fluctuations and errors commonly consists of monitoring an observable (temperature, blood-glucose level, etc.) and reacting on its controllers (heater power, insulin amount, etc.). In the quantum domain, there is a change of paradigm in feedback, since measurements modify the state of the system, most dramatically when the trajectory goes through superpositions of measurement eigenstates. Here, we demonstrate the stabilization of an arbitrary trajectory of a superconducting qubit by measurement-based feedback. The protocol benefits from the long coherence time $\left(T_{2}>10 \mu \mathrm{s}\right)$ of the 3D transmon qubit, the high efficiency $(82 \%)$ of the phasepreserving Josephson amplifier, and fast electronics that ensure less than $500 \mathrm{~ns}$ total delay. At discrete time intervals, the state of the qubit is measured and corrected in case an error is detected. For Rabi oscillations, where the discrete measurements occur when the qubit is supposed to be in the measurement pointer states, we demonstrate an average fidelity of $85 \%$ to the targeted trajectory. For Ramsey oscillations, which do not go through pointer states, the average fidelity reaches $76 \%$. Incidentally, we demonstrate a fast reset protocol that allows us to cool a $3 \mathrm{D}$ transmon qubit down to $0.6 \%$ in the excited state.
\end{abstract}

DOI: 10.1103/PhysRevX.3.021008

\section{INTRODUCTION}

The coupling of a quantum object to an environment is essential to enable its observation and manipulation. Yet, the mere existence of this coupling induces decoherence toward pointer states that are stable under monitoring of the environment [1]. There is thus a limiting time scale for the faithful preparation of a qubit in an arbitrary state or its control along a given trajectory in Hilbert space. As a part of the environment, an observer extracts information on the object and contributes to this time scale. However, if the observer acquires information faster than the uncontrolled part of the environment, it is possible to use it through a feedback process and permanently stabilize a given trajectory or state [2-5]. Superconducting qubits in cavities offer a test bed for these concepts, as well as good candidates for practical applications [6,7]. Recently, persistent Rabi oscillations have been demonstrated via analog measurement-based feedback using continuous weak measurement of a qubit [8], and qubit reset via digital measurement-based feedback using projective measurements has been performed [9]. In this work, we demonstrate a simple protocol to stabilize any trajectory of a single qubit by using a

Published by the American Physical Society under the terms of the Creative Commons Attribution 3.0 License. Further distribution of this work must maintain attribution to the author(s) and the published article's title, journal citation, and DOI.
Subject Areas: Condensed Matter Physics, Quantum Physics, Quantum Information stroboscopic digital feedback based on strong measurement [10]. During the manipulation of the qubit, its state is measured in a nearly projective manner at specific time intervals and a correcting control sequence is triggered conditionally on the outcome, so as to correct its trajectory from the errors that occur due to decoherence and relaxation. The efficiency of the trajectory stabilization relies on the rapidity to measure and react, compared to decoherence. In order to minimize these time scales, we use a phase-preserving quantum-limited amplifier (see Fig. 1) [11-13] and a field-programmable gate array (FPGA), adding a delay of only $360 \mathrm{~ns}$ when outputting a drive pulse that is conditioned on readout (see the Supplemental Material [14] and references therein [15-19]).

\section{FAST AND NONDEMOLITION PROJECTIVE MEASUREMENT}

The superconducting qubit follows the design of the 3D transmon developed in Ref. [20]. A single aluminum Josephson junction, connected to two antennas of $0.4 \mathrm{~mm}$ by $1 \mathrm{~mm}$ each, on a sapphire substrate, is embedded in an empty bulk aluminum cavity, whose first coupled modes are at $\omega_{c} / 2 \pi=7.748$ and $13 \mathrm{GHz}$ when the qubit is in its ground state. External coupling rates to the first mode $\kappa_{\text {in }} / 2 \pi=0.34 \mathrm{MHz}$ and $\kappa_{\text {out }} / 2 \pi=1.49 \mathrm{MHz}$ are chosen to be of the same order of magnitude as the inverse feedback delay (500 ns), and internal losses are negligible 

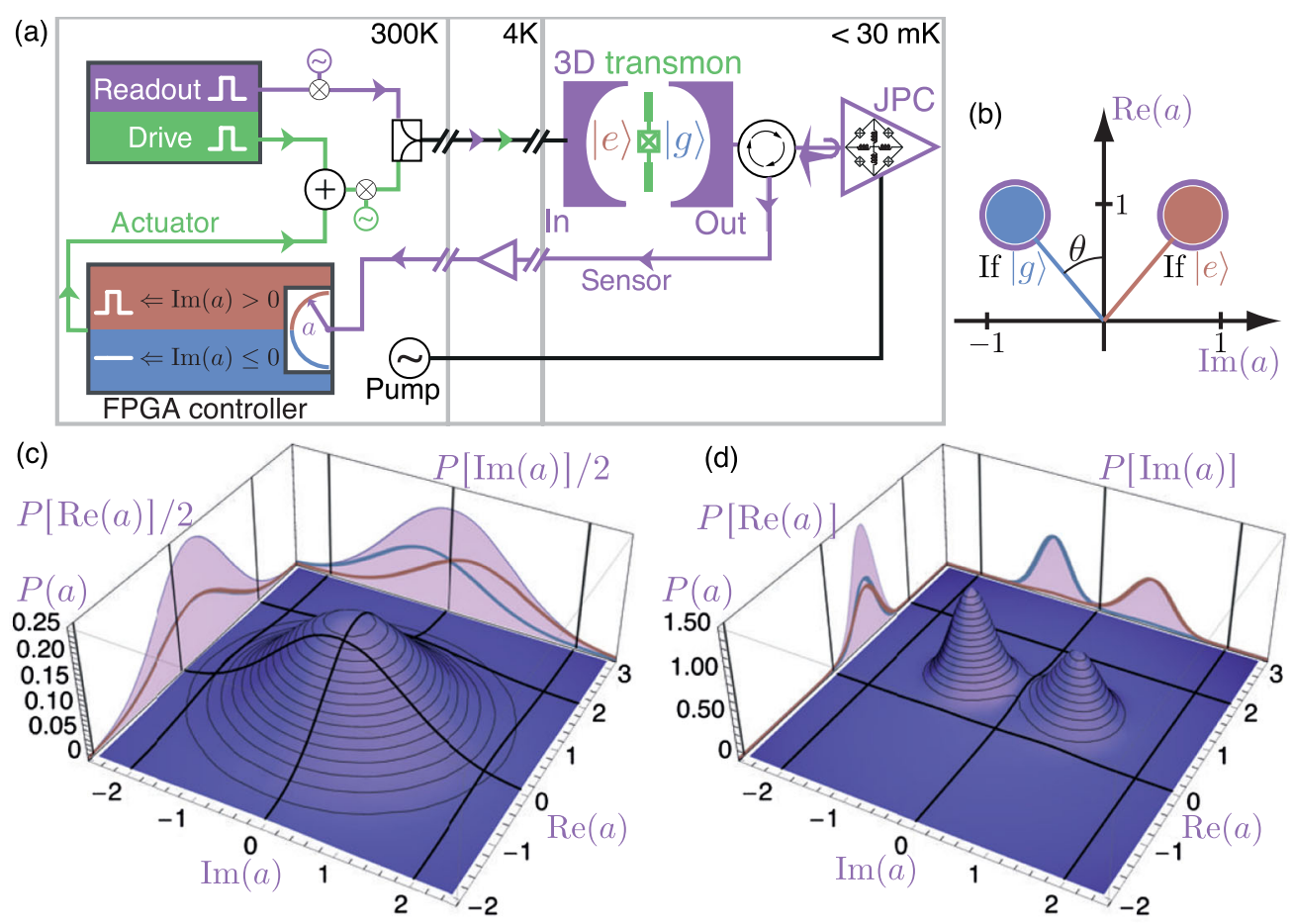

FIG. 1. (a) Schematics of the experiment. The state of a 3D transmon qubit is entangled with the phase of a coherent field transmitted through the cavity at frequency $\omega_{r}=\omega_{c}-\chi$. It is amplified by a Josephson parametric converter (JPC), and its complex amplitude $a$ is measured and averaged by digital demodulation using an FPGA board (sensing and control). The drive at qubit transition frequency $\omega_{e g}$ is modulated by the sum of a predetermined waveform and of a conditional one generated by the FPGA board (actuation). If the transmission measurement points toward state $|e\rangle$, the actuator generates a $\pi$ pulse to get the qubit back in $|g\rangle$. (b) Expected complex amplitude of the field in the cavity averaged over the measurement time $T_{\text {meas }}$ represented as the rod of a lollypop in the Fresnel space for both qubit states. The typical deviation due to vacuum fluctuations of the field in the $N_{\mathrm{m}}=11$ averaged modes is represented by the lollypop radius. The limited measurement efficiency $(\eta=67 \%)$ only slightly increases the observed deviations by $\eta^{-1 / 2}-1=22 \%$ (purple rings). (c),(d) Probability density with the JPC (c) off and (d) on, extracted from $10^{6}$ measurement outcomes when the qubit is prepared in state $|g\rangle$ or $|e\rangle$ with equal probability. Each outcome is the complex averaged amplitude of the field inside the transmon cavity at $\omega_{r}$. The halved probability density that corresponds to the preparation of $|g\rangle$ only (respectively, $|e\rangle$ ) is plotted in blue (orange), together with the projections along the real and imaginary axes. Turning on the pump of the amplifier as in (d) results in a great enhancement of the measurement fidelity, compared to the case without (c).

on these scales. The cavity is anchored to a dilution fridge below $30 \mathrm{mK}$ [14]. Spectroscopic measurements give a qubit frequency $\omega_{e g}=\omega_{c}-\Delta=2 \pi \times 3.576 \mathrm{GHz}$ that differs from the next transition by an anharmonicity $\left(\omega_{e g}-\omega_{f e}\right) / 2 \pi=198 \mathrm{MHz}$. The relaxation time $T_{1}=$ $28 \mu$ s corresponds to the Purcell limit [21], and the pure dephasing time is $T_{\phi}=14.5 \mu \mathrm{s}$ [Fig. 2(a)].

In the dispersive limit $\omega_{e g}-\omega_{f e} \ll \Delta$ [22], the cavity resonance frequency decreases by $2 \chi$ when the qubit goes from the ground to the excited state, and the dispersive shift here is $\chi / 2 \pi=0.78 \mathrm{MHz}$. The transmission measurement is strongest at readout frequency $\omega_{r}=\omega_{c}-\chi$, which minimizes the overlap between the two coherent states that correspond to the $|g\rangle$ and $|e\rangle$ qubit states (Fig. 1). In the experiment, square measurement pulses of $1.2 \mu \mathrm{s}$ are sent through the cavity. The amplitude of the readout field inside the cavity can be calibrated from the measurement-induced dephasing as a function of readout power, leading to 1.4 photons, on average [14]. The outgoing signal is amplified during these $1.2 \mu \mathrm{s}$ by using a Josephson parametric converter (JPC) [11-13] with $22 \mathrm{~dB}$ of gain over $6 \mathrm{MHz}$ (Fig. 1 and Ref. [14]) and following amplifiers before being down-converted and digitalized by using the FPGA board input. Note that the JPC is only turned on during measurement periods, so as to minimize decoherence that occurs due to backaction [14]. The board averages numerically both quadratures of the signal during the steady part of the outgoing pulse only [see Fig. 3(a)], which corresponds to about $N_{\mathrm{m}}=T_{\text {meas }}\left(\kappa_{\text {in }}+\kappa_{\text {out }}\right)=11$ temporal modes of 1.4 photons. States $|g\rangle$ and $|e\rangle$ for the qubit lead to two almost nonoverlapping coherent states for the average intracavity fields $\left|\alpha e^{-i \theta}\right\rangle$ and $\left|\alpha e^{i \theta}\right\rangle$, with $\theta \approx 40^{\circ}$ as expected from $\tan (\theta)=2 \chi /\left(\kappa_{\text {in }}+\kappa_{\text {out }}\right)$ [Fig. 1(b)]. With an ideal setup measuring both quadratures of the average complex field $a$ in the cavity, the variance on $a$ 


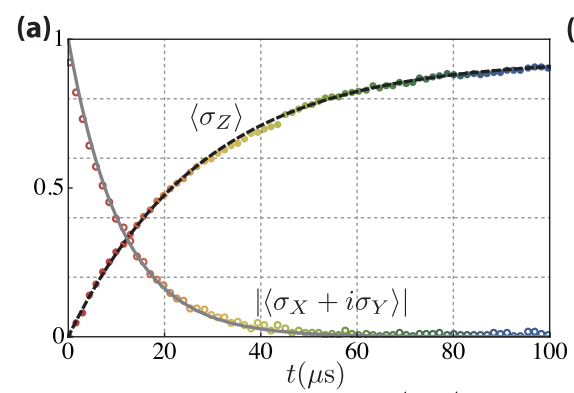

(b)
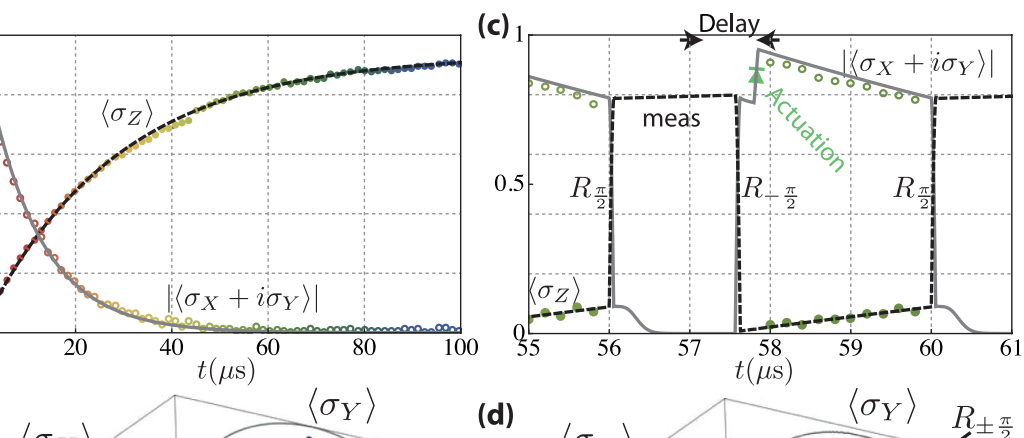

(d)

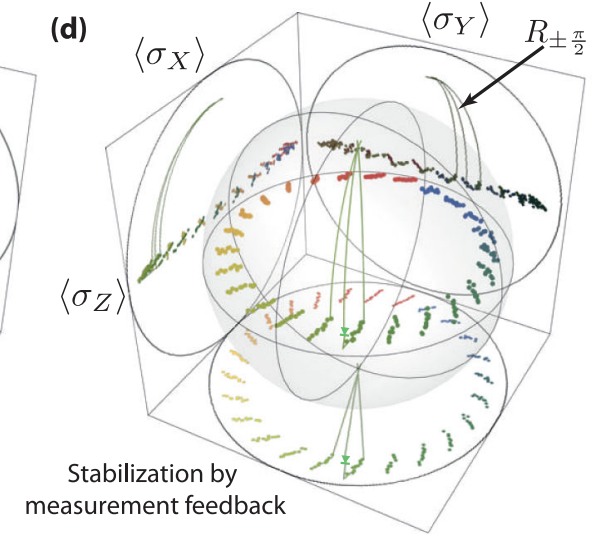

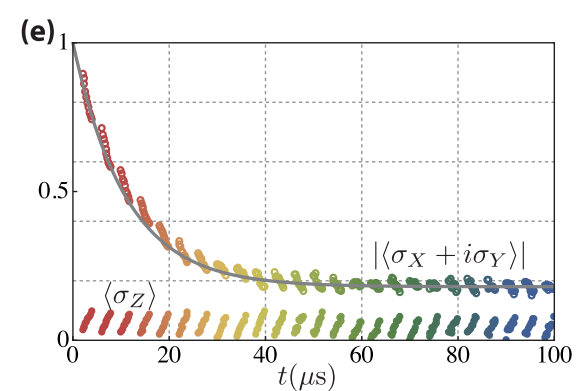

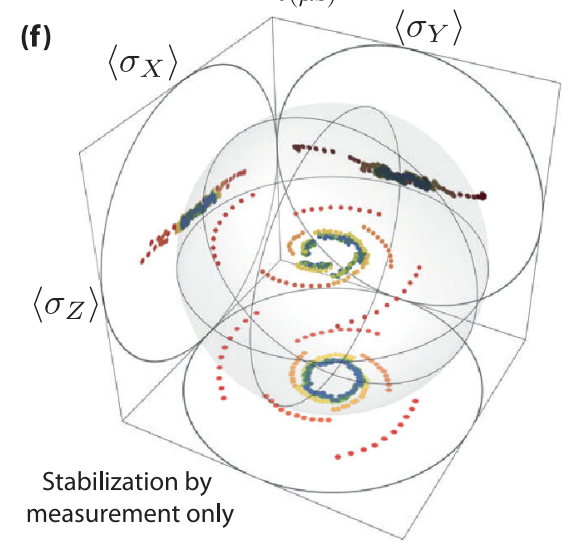

FIG. 2. (a) Evolution of $\left\langle\sigma_{Z}\right\rangle$ (filled dots) and of the coherence $\left|\left\langle\sigma_{X}+i \sigma_{Y}\right\rangle\right|$ (open circles) when the qubit is prepared in state $(|g\rangle+|e\rangle) / \sqrt{2}$ at time 0 . The color encodes the time identically in all panels. The lines are exponential fits that use coherence time $T_{2}=11.5 \mu \mathrm{s}$ and relaxation time $T_{1}=28 \mu \mathrm{s}$. (b) Same evolution represented in the Bloch sphere with a Ramsey frequency $\omega_{\mathrm{Ry}} / 2 \pi=100 \mathrm{kHz}$. At each time (color), the outcome of qubit tomography is represented as a filled dot in the Bloch sphere and in the three orthogonal projection planes. The large open black circles set the scale of the Bloch sphere extrema. (c) Same evolution as in (a), with stroboscopic measurement feedback every $4 \mu$ s. State tomography is only performed outside of the sensing and actuation periods. The lines represent the results of a simulation without extra fit parameters. When the conditional $\pi$ pulse occurs (actuation), the average purity increases so that the coherences are permanently preserved, on average. (d) Same evolution represented in the Bloch sphere with a Ramsey frequency $\omega_{\mathrm{Ry}} / 2 \pi=10 \mathrm{kHz}$, instead of $100 \mathrm{kHz}$ for a clearer observation of the trajectory. The simulated trajectory is represented as a line only for the time interval chosen in (c) for clarity. (e) Evolution of the qubit with the same process as in (c),(d) but without actuation. The exponential fit, using the same $T_{2}$ as in (a), indicates an average persistent coherence of $18 \%$ without any actuation. (f) In the Bloch sphere, the Ramsey frequency is chosen to be $\omega_{\mathrm{Ry}} / 2 \pi=100 \mathrm{kHz}$.

should be given by $1 / \sqrt{N_{\mathrm{m}}}$ [23]. In the experiment, the $19 \%$ loss of signal through the input of the cavity $\left(\kappa_{\text {in }} / \kappa_{\text {tot }}\right)$ and the efficiency of the detection setup $(82 \%)$ degrade the signal by only $67 \%$ beyond this variance (Fig. 1). Therefore, measuring $\operatorname{Im}(a)>0$ on the readout field indicates a qubit in the excited state $|e\rangle$ with a fidelity beyond $99.8 \%$, taking aside the expected false counts due to relaxation events during readout. All measurement pulses in this article are performed according to this procedure, and the $0.2 \%$ infidelity is neglected throughout. Using this setup, it is possible to perform almost projective and quantum nondemolition measurements of the qubit state much faster than decoherence [24,25], a crucial ingredient of measurement-based feedback. An illustration of the discriminating power of the setup is shown as a histogram of measurement outcomes (average complex amplitude in the cavity) with the JPC amplifier on or off [Figs. 1(c) and 1(d)] for a qubit starting randomly in state $|g\rangle$ or $|e\rangle$.

\section{COOLING A QUBIT USING MEASUREMENT- BASED FEEDBACK}

As a benchmark of our feedback hardware, we actively cool down the qubit to its ground state, similarly to what was demonstrated by Ristè et al. with a phase-sensitive amplifier and digital controller $[9,26]$. Quantum information processing requires such removal of entropy during initialization or when correcting for errors [27]. This method allows us to do so without fast frequency tuning [28-31], postselection [24,25], or limited coupling rate $\kappa<\chi$ [32]. An initial measurement determines the qubit state. If the outcome points toward the excited state $[\operatorname{Im}(a)>0]$, the FPGA controller emits a square pulse (Fig. 1), so as to apply a $\pi$ pulse around $Y$ on the qubit only $500 \mathrm{~ns}$ after the first readout pulse exits the cavity (see Ref. [14] for details). As an illustration, the qubit is first prepared in the most entropic mixed state $\rho=$ $(|g\rangle\langle g|+| e\rangle\langle e|) / 2$ by either applying a $\pi$ pulse or not, 

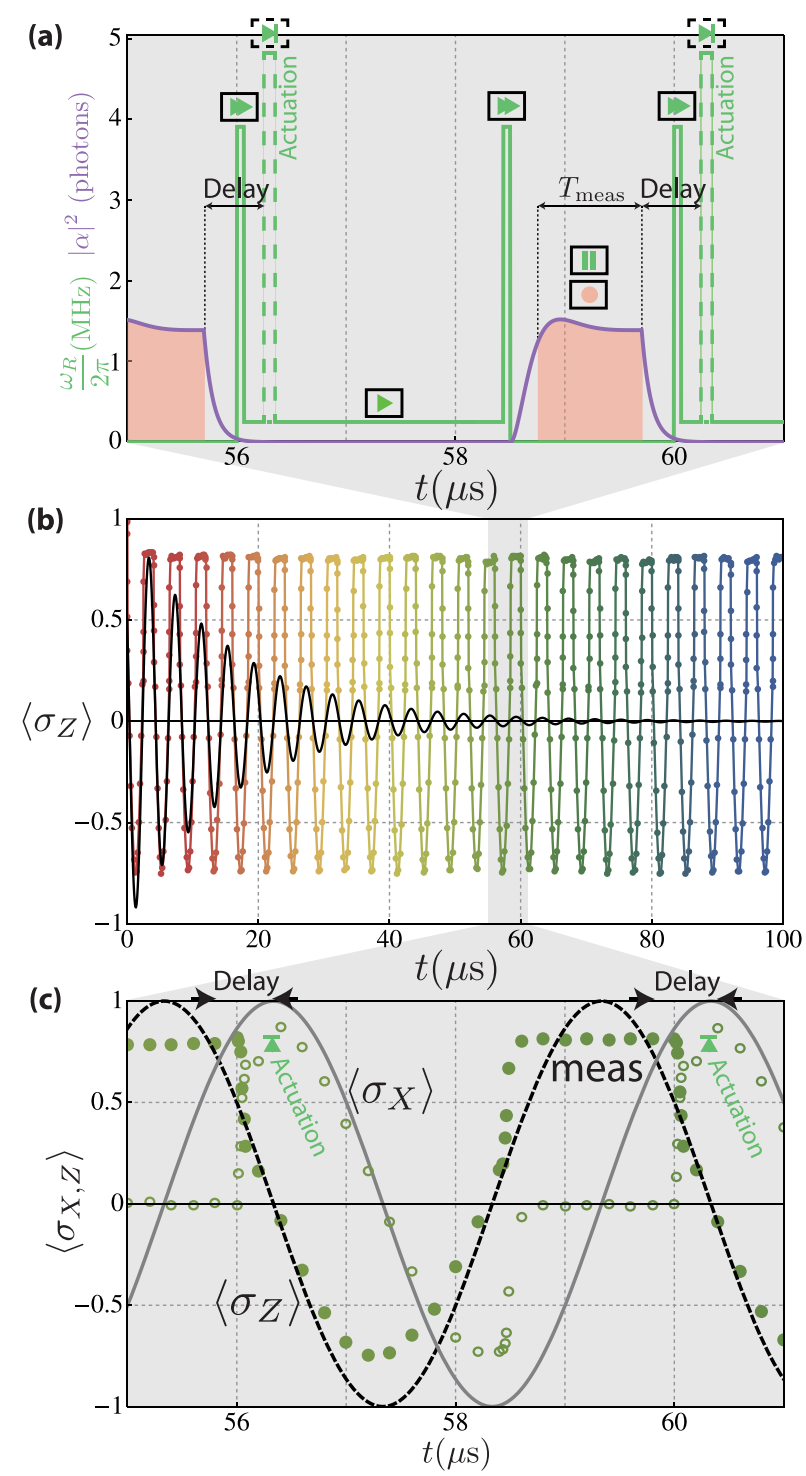

FIG. 3. (a) Pulse sequence for stabilizing Rabi oscillations. For a typical period of $4 \mu \mathrm{s}$, the green line represents the drive amplitude and the purple line the expected occupation of the cavity. The complex amplitude $a$ of the measurement field is recorded only during the steady part of the occupation (red areas). When $\operatorname{Im}(a)>0$, a fast $\pi$ pulse is applied after a total delay of $500 \mathrm{~ns}$ (actuation). These steps are illustrated with the usual symbols for a media player. (b) The black line represents the decaying Rabi oscillation around $\sigma_{Y}$ with frequency $\omega_{R}=250 \mathrm{kHz}$ and measured decay time $T_{R}=15.5 \mu \mathrm{s}$. The dots on the line represent persistent Rabi oscillations measured using the pulse sequence described in (a). (c) Same measurement as in (b) shown on a smaller span for $\left\langle\sigma_{Z}\right\rangle$ (filled dots) and $\left\langle\sigma_{X}\right\rangle$ (open circles). The targeted Rabi trajectory is shown as two lines, a dashed black line for $\left\langle\sigma_{Z}\right\rangle$ and a solid gray one for $\left\langle\sigma_{X}\right\rangle$.

and averaging the outcomes over these two possibilities. The probability $P_{|e\rangle}$ for the qubit to be in state $|e\rangle$ is then measured following zero, one, or more resets by feedback. We found that starting from $P_{|e\rangle}=50 \%$, a single reset
TABLE I. Error in the preparation of $|g\rangle$ using zero, one, or two resets by feedback when starting in the most entropic state or in the thermalized state (effectively at $46 \mathrm{mK}$ ).

\begin{tabular}{lccc}
\hline \hline Reset number & 0 & 1 & 2 \\
\hline From $(|g\rangle\langle g|+| e\rangle\langle e|) / 2$ & $50 \%$ & $3.6 \%$ & $1.1 \%$ \\
From thermalized state & $2.4 \%$ & $0.7 \%$ & $0.6 \%$ \\
\hline \hline
\end{tabular}

brings this level down to $P_{|e\rangle}=3.6 \%$, which would require it to thermalize during $110 \mu \mathrm{s}$ without feedback. Yet, events where the qubit relaxes between the middle of the measurement pulse and the feedback pulse limit the efficiency of a single reset. Doing a second reset immediately after the first brings the qubit much closer to the ground state with $P_{|e\rangle}=1.1 \%$. This value does not improve with additional feedback and is limited mostly by the excitation of higher qubit states during the first reset [9]. These higher states are almost empty $(0.06 \%)$ when starting from a thermalized qubit at $P_{|e\rangle}=2.4 \%$, and two consecutive resets by feedback cool the qubit further down to $P_{|e\rangle}=$ $0.6 \%$. These results are summarized in Table I. Note that this reset allows us to prepare any state with similar purity by using rotations of the qubit once the qubit is in state $|g\rangle$ and to increase the repetition rates of quantum algorithms [26].

\section{STABILIZING A QUANTUM TRAJECTORY USING STROBOSCOPIC FEEDBACK}

\section{A. Ramsey oscillations}

It is also possible to stabilize a state like $(|g\rangle+|e\rangle) / \sqrt{2}$, which is not an eigenstate of the measurement operator. First, a $\pi / 2$ pulse is applied to the qubit, so as to prepare it in $(|g\rangle+|e\rangle) / \sqrt{2}$ with a drive frequency $\omega_{\text {eg }}$. At any time $t$, it is possible to realize the full tomography of the qubit. Indeed, $\left\langle\sigma_{Z}\right\rangle$ is directly given by the average of the measurement outcomes, while $\left\langle\sigma_{X}\right\rangle$ (respectively, $\left\langle\sigma_{Y}\right\rangle$ ) is given by the same averaging, preceded by a rotation of the measurement axis using a 64-ns-long Rabi $\pi / 2$ pulse around $Y$ (respectively, $X$ ), where $\sigma_{X, Y, Z}$ are the Pauli matrices. In order to connect to the usual representation of Ramsey fringes at a given frequency $\omega_{\mathrm{Ry}}$, we can rotate the measurement axis linearly in time so that $\left\langle\sigma_{X}\right\rangle$ maps onto $\left\langle\cos \left(\omega_{\mathrm{Ry}} t\right) \sigma_{X}+\sin \left(\omega_{\mathrm{Ry}} t\right) \sigma_{Y}\right\rangle$ and $\left\langle\sigma_{Y}\right\rangle$ onto $\left\langle-\sin \left(\omega_{\mathrm{Ry}} t\right) \sigma_{X}+\cos \left(\omega_{\mathrm{Ry}} t\right) \sigma_{Y}\right\rangle$.

Without measurement-based feedback, the Bloch vector of the qubit decays exponentially both in $Z$ and in the $X, Y$ plane [Figs. 2(a) and 2(b)]. The decay in $Z$ is described by time scale $T_{1}=28 \mu \mathrm{s}$ while the decay in $X, Y$ is described by time scale $T_{2}=11.5 \mu \mathrm{s}$. In order to stabilize persistent Ramsey oscillations, a measurement of the qubit is performed after a $\pi / 2$ rotation every $4 \mu \mathrm{s}$. The rotation axis is chosen so that the measurement outcome should point to 
state $|g\rangle$ in the targeted trajectory and the qubit is rotated back to its original state by a $-\pi / 2$ pulse. Each time the qubit is found to be in the $|e\rangle$ state, the FPGA controller performs a fast $\pi$ pulse (actuation) with a delay of $500 \mathrm{~ns}$ after the measurement ends, which occurs after the $-\pi / 2$ pulse. Using this stroboscopic measurement-based feedback, Ramsey oscillations are indeed preserved indefinitely [Figs. 2(c) and 2(d)]. Using optical Bloch equations [14], one can calculate the predicted qubit trajectory that corresponds to this protocol [Fig. 2(c)], which is consistent with the experiment. Deviations from the experiment likely originate from the change in measurement-induced dephasing when the JPC is turned off. The average purity $\operatorname{Tr}\left(\rho^{2}\right)$ of the density matrix $\rho$ is calculated to be $85 \%$ from these simulations; the timeaveraged fidelity $F=\overline{\left\langle\psi_{\operatorname{targ}}|\rho(t)| \psi_{\text {targ }}\right\rangle}$ to the target trajectory $\left|\psi_{\text {targ }}\right\rangle=\left(|g\rangle+e^{i \omega_{\text {Ry }} t}|e\rangle\right) / \sqrt{2}$ is $F=76 \%$, and the average information quantity $1-\operatorname{Tr}(-\rho \log \rho)=0.60$ bit. Interestingly, the sole effect of stroboscopically measuring the qubit, without any measurement feedback, induces persistent Ramsey oscillations, except with less purity $(52 \%)$, fidelity (56\%), and information quantity (0.03 bit) [Figs. 2(e) and 2(f)]. This stabilization is due to the relaxation of the qubit during the measurement period toward state $|g\rangle$ that makes it more probable to reinitiate in state $(|g\rangle+|e\rangle) / \sqrt{2}$ than in state $(|g\rangle-|e\rangle) / \sqrt{2}$ after the measurement ends. It can be seen as a kind of reservoir engineering similar to Ref. [33], where the natural qubit decay is used as the dissipation source.

\section{B. Rabi oscillations}

In order to illustrate further the flexibility of stroboscopic projective measurement-based feedback, we have also stabilized Rabi oscillations. Although it is possible to perform this stabilization by using analog feedback on a weak, continuous measurement [8], we demonstrate here that discrete feedback events are more efficient [10]. Without feedback, a constant microwave signal at $\omega_{\text {eg }}$ induces a Rabi oscillation of the qubit around $\sigma_{Y}$ with decay time $T_{R}=15.5 \mu \mathrm{s}$ [Fig. 3(b)] and frequency set to $\omega_{R}=250 \mathrm{kHz}$. In order to make the Rabi oscillations persistent, a measurement is performed each time the qubit is supposed to be in state $|g\rangle$ [Fig. 3(a)]. The FPGA controller then sends a fast correcting $\pi$ pulse (actuation) at the qubit frequency $\omega_{e g}$ each time the measurement reveals that the qubit is in the excited state. In order to optimize the fidelity of the feedback-controlled trajectory to the targeted Rabi oscillation, the precession angle that is left idle during the measurement-with the Zeno effect freezing the trajectory anyway-is briefly accelerated before and after measurement to compensate exactly for that pause [see Fig. 3(a)]. As can be seen in Fig. 3(b), the Rabi oscillations are indeed stabilized permanently with this protocol. Their average fidelity to the targeted Rabi oscillation is $F=85 \%$, their average purity $80 \%$, and their average information quantity 0.50 bit. The discrete correction events lead to visible discontinuities in the trajectories, restoring the purity lost during the last Rabi period because of decoherence.

\section{CONCLUSION}

The differences between continuous [8] and stroboscopic measurement feedback are enlightening. Although both methods allow the stabilization of a dynamical quantum state, continuous measurement exerts a constant dephasing rate, while stroboscopic measurement allows variations of this rate in time. For trajectories like Rabi oscillations that go through eigenstates of the measurement observable (poles of the Bloch sphere), we benefit here from the versatility of stroboscopic feedback by measuring only close to state $|g\rangle$, which is insensitive to measurement-induced dephasing, hence better preserving coherence over the whole trajectory. Besides, the stroboscopic method enables us to stabilize trajectories like Ramsey oscillations, which never reach measurement eigenstates, by periodically rotating the measurement basis.

This work illustrates the possibilities offered by measurement-based feedback for circuit quantum electrodynamics in the case of a single qubit in a cavity. We have shown here that fast digital electronics combined with efficient detection allow us to realize elaborate quantum control protocols on these systems. Future error-correction codes will benefit from the malleability of a numerical approach where complex filters need to be used to protect a quantum algorithm from errors. Extending these protocols to multiqubit architectures should enable the preparation and stabilization of more complex entangled states and trajectories.

\section{ACKNOWLEDGMENTS}

We thank Hanhee Paik for discussions, Nathanaël Cottet for help with electromagnetic field simulations, and Jack Olejnik and Richard Pescari for realizing mechanical parts. Nanofabrication has been made within the consortium Salle Blanche Paris Centre and in the Quantronics Group, which we also thank. We thank the LERMA for slicing our sapphire wafers. This work was supported by the EMERGENCES Program Contract of Ville de Paris and by the ANR Contract No. ANR-12-JCJC-TIQS.

[1] W.H. Zurek, Decoherence, Einselection, and the Quantum Origins of the Classical, Rev. Mod. Phys. 75, 715 (2003).

[2] A. C. Doherty, S. Habib, K. Jacobs, H. Mabuchi, and S. M. Tan, Quantum Feedback Control and Classical Control Theory, Phys. Rev. A 62, 012105 (2000). 
[3] H. M. Wiseman and G. J. Milburn, Quantum Measurement and Control (Cambridge University Press, Cambridge, England, 2009).

[4] C. Sayrin, I. Dotsenko, X. Zhou, B. Peaudecerf, T. Rybarczyk, S. Gleyzes, P. Rouchon, M. Mirrahimi, H. Amini, M. Brune, J.-M. Raimond, and S. Haroche, Real-Time Quantum Feedback Prepares and Stabilizes Photon Number States, Nature (London) 477, 73 (2011).

[5] X. Zhou, I. Dotsenko, B. Peaudecerf, T. Rybarczyk, C. Sayrin, S. Gleyzes, J. M. Raimond, M. Brune, and S. Haroche, Field Locked to a Fock State by Quantum Feedback with Single Photon Corrections, Phys. Rev. Lett. 108, 243602 (2012).

[6] M.H. Devoret and J.M. Martinis, Implementing Qubits with Superconducting Integrated Circuits, Quantum Inf. Process. 3, 163 (2004).

[7] J. Clarke and F. K. Wilhelm, Superconducting Quantum Bits, Nature (London) 453, 1031 (2008).

[8] R. Vijay, C. Macklin, D. H. Slichter, S. J. Weber, K. W. Murch, R. Naik, A. N. Korotkov, and I. Siddiqi, Stabilizing Rabi Oscillations in a Superconducting Qubit Using Quantum Feedback, Nature (London) 490, 77 (2012).

[9] D. Ristè, C. C. Bultink, K. W. Lehnert, and L. DiCarlo, Feedback Control of a Solid-State Qubit Using HighFidelity Projective Measurement, Phys. Rev. Lett. 109, 240502 (2012).

[10] M. Mirrahimi, B. Huard, and M. Devoret, in Proceedings of the 51st IEEE Conference on Decision and Control, 2012 (IEEE, New York, 2012).

[11] N. Bergeal, R. Vijay, V.E. Manucharyan, I. Siddiqi, R. J. Schoelkopf, S. M. Girvin, and M.H. Devoret, Analog Information Processing at the Quantum Limit with a Josephson Ring Modulator, Nat. Phys. 6, 296 (2010).

[12] N. Bergeal, F. Schackert, M. Metcalfe, R. Vijay, V.E. Manucharyan, L. Frunzio, D. E. Prober, R. J. Schoelkopf, S. M. Girvin, and M.H. Devoret, PhasePreserving Amplification near the Quantum Limit with a Josephson Ring Modulator, Nature (London) 465, 64 (2010).

[13] N. Roch, E. Flurin, F. Nguyen, P. Morfin, P. CampagneIbarcq, M. H. Devoret, and B. Huard, Widely Tunable, Nondegenerate Three-Wave Mixing Microwave Device Operating near the Quantum Limit, Phys. Rev. Lett. 108, 147701 (2012).

[14] See Supplemental Material at http://link.aps.org/ supplemental/10.1103/PhysRevX.3.021008 for technical details, calibration procedures, and simulation protocols.

[15] R. Barends et al., Minimizing Quasiparticle Generation from Stray Infrared Light in Superconducting Quantum Circuits, Appl. Phys. Lett. 99, 113507 (2011).

[16] A. D. Córcoles, J.M. Chow, J.M. Gambetta, C. Rigetti, J. R. Rozen, G. A. Keefe, M. B. Rothwell, M. B. Ketchen, and M. Steffen, Protecting Superconducting Qubits from Radiation, Appl. Phys. Lett. 99, 181906 (2011).

[17] D. I. Schuster, A. Wallraff, A. Blais, L. Frunzio, R.-S. Huang, J. Majer, S. M. Girvin, and R. J. Schoelkopf, ac Stark Shift and Dephasing of a Superconducting
Qubit Strongly Coupled to a Cavity Field, Phys. Rev. Lett. 94, 123602 (2005).

[18] F. R. Ong, M. Boissonneault, F. Mallet, A. Palacios-Laloy, A. Dewes, A. C. Doherty, A. Blais, P. Bertet, D. Vion, and D. Esteve, Circuit QED with a Nonlinear Resonator: ac-Stark Shift and Dephasing, Phys. Rev. Lett. 106, 167002 (2011).

[19] J. Gambetta, A. Blais, D. I. Schuster, A. Wallraff, L. Frunzio, J. Majer, M. H. Devoret, S. M. Girvin, and R. J. Schoelkopf, Qubit-Photon Interactions in a Cavity: Measurement-Induced Dephasing and Number Splitting, Phys. Rev. A 74, 042318 (2006).

[20] H. Paik, D. I. Schuster, L. S. Bishop, G. Kirchmair, G. Catelani, A.P. Sears, B. R. Johnson, M.J. Reagor, L. Frunzio, L. I. Glazman, S. M. Girvin, M. H. Devoret, and R. J. Schoelkopf, Observation of High Coherence in Josephson Junction Qubits Measured in a ThreeDimensional Circuit QED Architecture, Phys. Rev. Lett. 107, 240501 (2011).

[21] A. A. Houck, J. A. Schreier, B. R. Johnson, J. M. Chow, J. Koch, J.M. Gambetta, D. I. Schuster, L. Frunzio, M.H. Devoret, S.M. Girvin, and R. J. Schoelkopf, Controlling the Spontaneous Emission of a Superconducting Transmon Qubit, Phys. Rev. Lett. 101, 080502 (2008).

[22] S. E. Nigg, H. Paik, B. Vlastakis, G. Kirchmair, S. Shankar, L. Frunzio, M.H. Devoret, R. J. Schoelkopf, and S.M. Girvin, Black-Box Superconducting Circuit Quantization, Phys. Rev. Lett. 108, 240502 (2012).

[23] C. M. Caves, J. Combes, Z. Jiang, and S. Pandey, Quantum Limits on Phase-Preserving Linear Amplifiers, Phys. Rev. A 86, 063802 (2012).

[24] J. E. Johnson, C. Macklin, D. H. Slichter, R. Vijay, E. B. Weingarten, J. Clarke, and I. Siddiqi, Heralded State Preparation in a Superconducting Qubit, Phys. Rev. Lett. 109, 050506 (2012).

[25] D. Ristè, J. G. van Leeuwen, H.-S. Ku, K. W. Lehnert, and L. DiCarlo, Initialization by Measurement of $a$ Superconducting Quantum Bit Circuit, Phys. Rev. Lett. 109, 050507 (2012).

[26] D. Ristè, C.C. Bultink, M. J. Tiggelman, R. N. Schouten, K. W. Lehnert, and L. DiCarlo, Millisecond Charge-Parity Fluctuations and Induced Decoherence in a Superconducting Qubit, arXiv:1212.5459v1.

[27] D.P. DiVincenzo, The Physical Implementation of Quantum Computation, Fortschr. Phys. 48, 771 (2000).

[28] S. O. Valenzuela, W.D. Oliver, D. M. Berns, K. K. Berggren, L.S. Levitov, and T.P. Orlando, MicrowaveInduced Cooling of a Superconducting Qubit, Science 314, 1589 (2006).

[29] M. Grajcar, S. H. W. van der Ploeg, A. Izmalkov, E. Il'ichev, H.-G. Meyer, A. Fedorov, A. Shnirman, and G. Schön, Sisyphus Cooling and Amplification by a Superconducting Qubit, Nat. Phys. 4, 612 (2008).

[30] M. D. Reed, B. R. Johnson, A. A. Houck, L. DiCarlo, J. M. Chow, D. I. Schuster, L. Frunzio, and R. J. Schoelkopf, Fast Reset and Suppressing Spontaneous Emission of a Superconducting Qubit, Appl. Phys. Lett. 96, 203110 (2010).

[31] M. Mariantoni, H. Wang, T. Yamamoto, M. Neeley, R. C. Bialczak, Y. Chen, M. Lenander, E. Lucero, 
A. D. O'Connell, D. Sank, M. Weides, J. Wenner, Y. Yin, J. Zhao, A. N. Korotkov, A. N. Cleland, and J. M. Martinis, Implementing the Quantum von Neumann Architecture with Superconducting Circuits, Science 334, 61 (2011).

[32] K. Geerlings, Z. Leghtas, I. M. Pop, S. Shankar, L. Frunzio, R. J. Schoelkopf, M. Mirrahimi, and M.H. Devoret,
Demonstrating a Driven Reset Protocol for a Superconducting Qubit, Phys. Rev. Lett. 110, 120501 (2013).

[33] K. W. Murch, U. Vool, D. Zhou, S. J. Weber, S. M. Girvin, and I. Siddiqi, Cavity-Assisted Quantum Bath Engineering, Phys. Rev. Lett. 109, 183602 (2012). 